Sevdzhan Hakkaev (Shumen)

\title{
SCATTERING OF SMALL SOLUTIONS OF A SYMMETRIC REGULARIZED-LONG-WAVE EQUATION
}

Abstract. We study the decay in time of solutions of a symmetric regularized-long-wave equation and we show that under some restriction on the form of nonlinearity, the solutions of the nonlinear equation have the same long time behavior as those of the linear equation. This behavior allows us to establish a nonlinear scattering result for small perturbations.

1. Introduction. In this paper we study the asymptotic behavior in time and scattering of small solutions of the following symmetric regularizedlong-wave equation (SRLW):

$$
u_{t t}-u_{x x}+f(u)_{x t}-u_{x x t t}=0
$$

or, equivalently, the system of equations

$$
\left\{\begin{array}{l}
u_{t}-u_{x x t}+f(u)_{x}-v_{x}=0, \\
v_{t}-u_{x}=0
\end{array}\right.
$$

This is a model that describes weakly nonlinear ion acoustic and spacecharge waves [7]. The SRLW equation is obviously symmetric in $t$ and $x$ derivatives and is very similar to the regularized-long-wave equation (RLW) which describes unidirectional propagation of nonlinear dispersive waves,

$$
u_{t}+u_{x}-u_{x x t}+f(u)_{x}=0 .
$$

In many nonlinear dispersive equations, solitary waves play an important role, and criteria for stability and instability are often related in some way to well-posedness and blow up questions. For equation (1.3) with $f(u)=u^{p+1}$ all solitary waves are stable when $p \leq 4$, and when $p>4$, there is a critical value $c_{r}>1$ such that a solitary wave is stable for wave speed $c>c_{r}$ and

2000 Mathematics Subject Classification: 35B40, 35Q35, 76B15.

Key words and phrases: asymptotic behavior of solutions, scattering theory.

Partially supported by Grant MM of MESC and by Scientific Research Grant 26/01.04.2004 of Shumen University. 
unstable for $1<c \leq c_{r}$ [8]. It is known that for sufficiently small initial data and suitable $p$ the solutions of (1.3) tend to 0 as $t \rightarrow \infty$ with an algebraic decay rate $t^{-1 / 3}$.

For equation (1.1), when the nonlinearity has the special form $f(u)=u^{p}$ and $p \leq 5$, all solitary waves are stable, and when $p>5$, there is a critical value $c_{0}$ such that a solitary wave is stable for $c>c_{0}$ and unstable for $1<c<c_{0}$.

Our aim is to describe the asymptotics of solutions of (1.2) in the case of nonlinearity of the form $f(u)=u^{p+1}$. The estimates of solutions obtained below guarantee that small solutions of the nonlinear problem behave asymptotically like solutions of the associated linear problem. Hence, this behavior allows us to establish a nonlinear scattering result.

Throughout this paper we use the notations $|\cdot|_{p}$ for the norm in the space $L^{p}(\mathbb{R})$ with $1 \leq p<\infty,\|\cdot\|_{s}$ for the norm in the Sobolev space $H^{s}(\mathbb{R}),\|\cdot\|_{0}=|\cdot|_{2}$, and we equip $X^{s}=H^{s}(\mathbb{R}) \times H^{s-1}(\mathbb{R})$ with the norm $\|\vec{u}\|_{X^{s}}=\|(u, v)\|_{X^{s}}=\|u\|_{s}+\|v\|_{s-1}$. We define $\Lambda^{s}=\left(1-\partial_{x}^{2}\right)^{s / 2}$ for any $s \geq 0$.

2. Preliminary results. In this section we present several lemmas which are needed to obtain our main results. First we will discuss the global existence and regularity of solutions of (1.2).

Theorem 2.1 (Global existence). Let $\vec{u}_{0}=\left(u_{0}, v_{0}\right) \in X^{1}$ and $f$ : $\mathbb{R} \rightarrow \mathbb{R}$ be a $C^{1}$ function with $f(0)=0$. Then there exists a unique solution $\vec{u}=(u, v)$ of $(1.2)$ in $C\left([0, \infty) ; X^{1}\right)$ with $\vec{u}(0)=\vec{u}_{0}$.

Proof. For the proof, see [4].

The group of linear operators associated with the linear system

$$
\left\{\begin{array}{l}
u_{t}-u_{x x t}-v_{x}=0 \\
v_{t}-u_{x}=0
\end{array}\right.
$$

will be denoted by $S(t)$, so $S(t) \vec{u}_{0}$ solves $(2.1)$ with initial data $\vec{u}(0)=\vec{u}_{0}$. Using the Fourier transform we can write (2.1) in the form

$$
\frac{d}{d t}\left(\begin{array}{c}
\widehat{u} \\
\widehat{v}
\end{array}\right)+i k A(k)\left(\begin{array}{c}
\widehat{u} \\
\widehat{v}
\end{array}\right)=0,
$$

where $A(k)=\left(\begin{array}{cc}0 & -\frac{1}{1+k^{2}} \\ -1 & 0\end{array}\right)$, since the system (2.1) is equivalent to

$$
\left\{\begin{array}{l}
u_{t}-\left(1-\partial_{x}^{2}\right)^{-1} \partial_{x} v=0 \\
v_{t}-u_{x}=0
\end{array}\right.
$$

The formal solution of $(2.2)$ with initial data $\left(\widehat{u}_{0}, \widehat{v}_{0}\right)$ is

$$
\left(\begin{array}{l}
\widehat{u}(\xi, t) \\
\widehat{v}(\xi, t)
\end{array}\right)=e^{-i \xi A(\xi)}\left(\begin{array}{c}
\widehat{u}_{0} \\
\widehat{v}_{0}
\end{array}\right)
$$


and a straightforward computation shows that

$$
e^{-i \xi A(\xi) t}=\left(\begin{array}{cc}
\cos \left(\frac{\xi t}{\sqrt{1+\xi^{2}}}\right) & \frac{i}{\sqrt{1+\xi^{2}}} \sin \left(\frac{\xi t}{\sqrt{1+\xi^{2}}}\right) \\
i \sqrt{1+\xi^{2}} \sin \left(\frac{\xi t}{\sqrt{1+\xi^{2}}}\right) & \cos \left(\frac{\xi t}{\sqrt{1+\xi^{2}}}\right)
\end{array}\right) .
$$

The solution of (1.2) satisfies the Duhamel formula

$$
\vec{u}(x, t)=S(t) \vec{u}_{0}(x)+\int_{0}^{t} S(t-\tau) \partial_{x}\left(\begin{array}{c}
-\Lambda^{-2} f(u(\tau)) \\
0
\end{array}\right) d \tau .
$$

We have the following lemma (improving results in [4]).

Lemma 2.1. For $n, t>1$ and $\varepsilon<1$,

$$
\sup _{\alpha \in \mathbb{R}}\left|\int_{|\xi|<n} e^{i t h(\xi, \alpha)} d \xi\right| \leq c\left(\varepsilon+t^{-1 / 2} \max \left\{\varepsilon^{-2}, n^{2}\right\}\right),
$$

where $h(\xi, \alpha)=\xi / \sqrt{1+\xi^{2}}+\alpha \xi$ and $c$ is a constant.

The proof of Lemma 2.1 is based on the van der Corput lemma and can be found in $[6]$.

The next lemma concerns the decay of solutions of the linear system (2.1). The estimate obtained in the lemma allows us to prove our main result, namely the decay of solutions of (1.2).

LEMMA 2.2. Let $\vec{u}(t)=(u(t), v(t))$ be a solution of the linear system (2.1) and $\vec{u}_{0} \in X^{s+1}, \Lambda^{1} u_{0} \in L^{1}(\mathbb{R}), v_{0} \in L^{1}(\mathbb{R})$. Then

$$
|\vec{u}|_{\infty} \leq c_{0}\left(\left\|\vec{u}_{0}\right\|_{X^{s+1}}+\left|v_{0}\right|_{1}+\left|\Lambda^{1} u_{0}\right|_{1}\right)(1+t)^{-\theta}
$$

where $s>1 / 2$ and

$$
\theta= \begin{cases}\frac{1}{2 s+3}, & s \geq 3 / 2, \\ \frac{2 s-1}{2(2 s+3)}, & s \leq 3 / 2,\end{cases}
$$

and $c_{0}$ is a constant depending only on $s$.

Proof. Since

$$
\begin{aligned}
\vec{u}(t) & =S(t) \vec{u}_{0} \\
& =\int_{-\infty}^{\infty} e^{i x \xi}\left(\begin{array}{cc}
\cos \left(\frac{\xi t}{\sqrt{1+\xi^{2}}}\right) & \frac{i}{\sqrt{1+\xi^{2}}} \sin \left(\frac{\xi t}{\sqrt{1+\xi^{2}}}\right) \\
i \sqrt{1+\xi^{2}} \sin \left(\frac{\xi t}{\sqrt{1+\xi^{2}}}\right) & \cos \left(\frac{\xi t}{\sqrt{1+\xi^{2}}}\right)
\end{array}\right) \widehat{\overrightarrow{\vec{u}}}_{0}(\xi) d \xi
\end{aligned}
$$

we obtain 


$$
\begin{aligned}
|\vec{u}(t)| \leq & \frac{1}{4 \pi} \sum\left|\int_{-\infty}^{\infty}\left(\widehat{u}_{0} \pm \frac{1}{\sqrt{1+\xi^{2}}} \widehat{v}_{0}\right) e^{i t\left( \pm \xi / \sqrt{1+\xi^{2}}+x \xi / t\right)} d \xi\right| \\
& +\frac{1}{4 \pi} \sum\left|\int_{-\infty}^{\infty}\left(\widehat{v}_{0} \pm \sqrt{1+\xi^{2}} \widehat{u}_{0}\right) e^{i t\left( \pm \xi / \sqrt{1+\xi^{2}}+x \xi / t\right)} d \xi\right| \\
\leq & \frac{1}{4 \pi} \sum\left|\int_{-\infty}^{\infty}\left(\widehat{u}_{0} \pm \widehat{\Lambda^{-1}} v_{0}\right) e^{i t\left( \pm \xi / \sqrt{1+\xi^{2}}+x \xi / t\right)} d \xi\right| \\
& +\frac{1}{4 \pi} \sum\left|\int_{-\infty}^{\infty}\left(\widehat{v}_{0} \pm \widehat{\Lambda^{1}} u_{0}\right) e^{i t\left( \pm \xi / \sqrt{1+\xi^{2}}+x \xi / t\right)} d \xi\right| \\
\leq & \frac{1}{2 \pi} \int_{|\xi|>n}\left(\left|\widehat{u}_{0}\right|+\left|\widehat{\Lambda^{-1}} v_{0}\right|+\left|\widehat{v}_{0}\right|+\left|\widehat{\Lambda^{1} u_{0}}\right|\right) d \xi \\
& +\frac{1}{4 \pi} \sum\left|\int_{-\infty}^{\infty}\left(u_{0}(y) \pm \Lambda^{-1} v_{0}(y)\right) d y\right| \cdot\left|\int_{-n}^{n} e^{i t\left( \pm \xi / \sqrt{1+\xi^{2}}+x \xi / t\right)} d \xi\right| \\
& +\frac{1}{4 \pi} \sum\left|\int_{-\infty}^{\infty}\left(v_{0}(y) \pm \Lambda^{1} u_{0}(y)\right) d y\right| \cdot\left|\int_{-n}^{n} e^{i t\left( \pm \xi / \sqrt{1+\xi^{2}}+x \xi / t\right)} d \xi\right|
\end{aligned}
$$

where the sums are over the two choices of sign. Hence

$$
\begin{aligned}
|\vec{u}(t)| \leq & \left(\left\|u_{0}\right\|_{s}+\left\|v_{0}\right\|_{s}+\left\|\Lambda^{1} u_{0}\right\|_{s}+\left\|\Lambda^{-1} v_{0}\right\|_{s}\right)\left(\int_{|\xi| \geq n}\left(1+\xi^{2}\right)^{-s} d \xi\right)^{1 / 2} \\
& +c\left(\varepsilon+t^{-1 / 2} \max \left\{\varepsilon^{-2}, n^{2}\right\}\right)\left(\left|\vec{u}_{0}\right|_{L^{1} \times L^{1}}+\left|\Lambda^{1} u_{0}\right|_{1}+\left|\Lambda^{-1} v_{0}\right|_{1}\right) \\
|\vec{u}(t)| \leq & \left(\left\|u_{0}\right\|_{s}+\left\|v_{0}\right\|_{s}+\left\|\Lambda^{1} u_{0}\right\|_{s}+\left\|\Lambda^{-1} v_{0}\right\|_{s}\right) n^{-(s-1 / 2)} \\
& +c\left(\varepsilon+t^{-1 / 2} \max \left\{\varepsilon^{-2}, n^{2}\right\}\right)\left(\left|\vec{u}_{0}\right|_{L^{1} \times L^{1}}+\left|\Lambda^{1} u_{0}\right|_{1}+\left|\Lambda^{-1} v_{0}\right|_{1}\right) .
\end{aligned}
$$

Choosing $\varepsilon=t^{-\alpha}$ and $n=t^{\alpha}$, we deduce

$$
\begin{aligned}
|\vec{u}(t)| \leq & \left(\left\|u_{0}\right\|_{s}+\left\|v_{0}\right\|_{s}+\left\|\Lambda^{1} u_{0}\right\|_{s}+\left\|\Lambda^{-1} v_{0}\right\|_{s}\right) t^{-\alpha(s-1 / 2)} \\
& +c\left(\left|u_{0}\right|_{1}+\left|v_{0}\right|_{1}+\left|\Lambda^{1} u_{0}\right|_{1}\right)\left(t^{-\alpha}+t^{-1 / 2} t^{2 \alpha}\right), \\
|\vec{u}(t)| \leq & \left(\left\|u_{0}\right\|_{s+1}+\left\|v_{0}\right\|_{s}\right) t^{-\alpha(s-1 / 2)} \\
& +c\left(\left|u_{0}\right|_{1}+\left|v_{0}\right|_{1}+\left|\Lambda^{1} u_{0}\right|_{1}\right)\left(t^{-\alpha}+t^{-1 / 2} t^{2 \alpha}\right) .
\end{aligned}
$$

Let $\alpha(s-1 / 2)=1 / 2-2 \alpha$. It follows that

$$
|\vec{u}(t)|_{\infty} \leq c\left(\left\|u_{0}\right\|_{s+1}+\left\|v_{0}\right\|_{s}+\left|u_{0}\right|_{1}+\left|v_{0}\right|_{1}+\left|\Lambda^{1} u_{0}\right|_{1}\right) t^{-\theta},
$$

where

$$
\theta= \begin{cases}\frac{1}{2 s+3}, & s \geq 3 / 2 \\ \frac{2 s-1}{2(2 s+3)}, & s \leq 3 / 2\end{cases}
$$




\section{Decay and scattering of solutions of the nonlinear equation}

THEOREM 3.1. Let $f(u)=u^{p+1}$ and $p>11$. Then there is a constant $\delta>0$ such that for any $\vec{u}_{0} \in X^{2}, \Lambda^{1} u_{0} \in L^{1}, v_{0} \in L^{1}$ for which $\left\|\vec{u}_{0}\right\|_{X^{2}}+$ $\left|\Lambda^{1} u_{0}\right|_{1}+\left|v_{0}\right|_{1}<\delta$, the solution $\vec{u}(x, t)$ of (1.2) satisfies

$$
|\vec{u}(x, t)| \leq C\left(\vec{u}_{0}\right)(1+t)^{-1 / 10}
$$

for all $t>0$ and $x \in \mathbb{R}$.

Proof. From the Duhamel formula

$$
\vec{u}(t)=S(t) \vec{u}_{0}+\int_{0}^{t} S(t-\tau) \partial_{x}\left(\begin{array}{c}
-\Lambda^{-2} f(u(\tau)) \\
0
\end{array}\right) d \tau
$$

and Lemma 2.2, for $s=1$ we obtain

$$
\begin{aligned}
(1+t)^{1 / 10}|\vec{u}(t)| & \leq\left|S(t) \vec{u}_{0}\right|+\int_{0}^{t}\left|S(t-\tau) \partial_{x}\left(\begin{array}{c}
-\Lambda^{-2} f(u(\tau)) \\
0
\end{array}\right)\right| d \tau \\
& \leq c\left(\left\|u_{0}\right\|_{2}+\left\|v_{0}\right\|_{1}+\left|v_{0}\right|_{1}+\left|\Lambda^{1} u_{0}\right|_{1}\right) \\
+c(1+t)^{1 / 10} & \int_{0}^{t}(1+t-\tau)^{-1 / 10}\left(\left\|\partial_{x} \Lambda^{-2} f(u)\right\|_{2}+\left|\Lambda^{1}\left(\partial_{x} \Lambda^{-2} f(u)\right)\right|_{1}\right) d t
\end{aligned}
$$

We have

$$
\begin{aligned}
\left\|\Lambda^{-2} \partial_{x} f(u)\right\|_{2} & =\int_{\mathbb{R}}\left(1+|\xi|^{2}\right)^{2} \frac{|\xi|^{2}}{\left(1+|\xi|^{2}\right)^{2}}|\widehat{f(u)}(\xi)|^{2} d \xi \\
& \leq c\|f(u)\|_{1} \leq c|u|_{\infty}^{p}\|u\|_{1} .
\end{aligned}
$$

Since the operator $\Lambda^{-1}$ is the convolution with a function from $L^{1}(\mathbb{R})$ (see Lemma 1.12 in [8]), it follows that

$$
\begin{aligned}
\left|\Lambda^{1}\left(\partial_{x} \Lambda^{-2} f(u)\right)\right|_{1} & =\left|\Lambda^{-1}\left(\partial_{x} f(u)\right)\right|_{1} \leq c_{1}|u|_{\infty}^{p-1}\left|u u_{x}\right|_{1} \\
& \leq c_{1}|u|_{\infty}^{p-1}|u|_{2}\left|u_{x}\right|_{2} \leq c_{2}|u|_{\infty}^{p-1}\|u\|_{1}^{2} .
\end{aligned}
$$

From the above inequalities, we have

$$
\begin{aligned}
& (1+t)^{1 / 10}|\vec{u}(t)|_{\infty} \leq c\left(\left\|u_{0}\right\|_{2}+\left\|v_{0}\right\|_{1}+\left|v_{0}\right|_{1}+\left|\Lambda^{1} u_{0}\right|_{1}\right) \\
& +c(1+t)^{1 / 10} \int_{0}^{t}(1+t-\tau)^{-1 / 10}\left(|u|_{\infty}^{p}\|u\|_{1}+|u|_{\infty}^{p-1}\|u\|_{1}^{2}\right) d \tau
\end{aligned}
$$

Define

$$
q(t)=\sup _{0 \leq \tau \leq t}\left((1+\tau)^{1 / 10}|\vec{u}(\tau)|_{\infty}+\|u(\tau)\|_{1}\right) .
$$


Then from (3.2),

$$
\begin{aligned}
& (1+t)^{1 / 10}|\vec{u}|_{\infty} \leq c\left(\left\|u_{0}\right\|_{2}+\left\|v_{0}\right\|_{1}+\left|v_{0}\right|_{1}+\left|\Lambda^{1} u_{0}\right|_{1}\right) \\
& +c(1+t)^{1 / 10} \int_{0}^{t}(1+t-\tau)^{-1 / 10}\left((1+\tau)^{-p / 10}+(1+\tau)^{-(p-1) / 10}\right) d \tau q^{p+1}(t) .
\end{aligned}
$$

Since $p>11$ the last integral is bounded by $(1+t)^{-1 / 10}$. Therefore

$$
(1+t)^{1 / 10}|\vec{u}|_{\infty} \leq c\left(\left\|u_{0}\right\|_{2}+\left\|v_{0}\right\|_{1}+\left|v_{0}\right|_{1}+\left|\Lambda^{1} u_{0}\right|_{1}\right)+q^{p+1}(t) .
$$

Next, from (3.1) and Lemma 2.2 we obtain

$$
\begin{aligned}
\|\vec{u}(t)\|_{X^{2}} & \leq\left\|S(t) \vec{u}_{0}\right\|_{X^{2}}+\int_{0}^{t}\left\|S(t-\tau) \partial_{x}\left(\begin{array}{c}
-\Lambda^{-2} f(u) \\
0
\end{array}\right)\right\|_{X^{2}} d \tau \\
& \leq c\left(\left\|u_{0}\right\|_{2}+\left\|v_{0}\right\|_{1}\right)+\int_{0}^{t}\left\|u^{p+1}\right\|_{1} d \tau \\
& \leq c\left(\left\|u_{0}\right\|_{2}+\left\|v_{0}\right\|_{1}\right)+\int_{0}^{t}|u|_{\infty}^{p}\|u\|_{1} d \tau \leq c\left(\left\|u_{0}\right\|_{2}+\left\|v_{0}\right\|_{1}\right)+q^{p+1}(t) .
\end{aligned}
$$

Combining (3.3) and the above inequality we obtain

$$
q(t) \leq A\left(\left\|u_{0}\right\|_{2}+\left\|v_{0}\right\|_{1}+\left|v_{0}\right|_{1}+\left|\Lambda^{1} u_{0}\right|_{1}+q^{p+1}(t)\right) .
$$

Choose a number $\eta>0$ such that $\eta>A \eta^{p+1}$, where $A$ is the same constant appearing in (3.4). Choose $\delta>0$ such that if

$$
\left\|\vec{u}_{0}\right\|_{X^{2}}+\left|\Lambda^{1} u_{0}\right|_{1}+\left|v_{0}\right|_{1}<\delta
$$

then $q(0)<\eta$ and

$$
\eta>A\left[\left\|\vec{u}_{0}\right\|_{X^{2}}+\left|\Lambda^{1} u_{0}\right|_{1}+\left|v_{0}\right|_{1}+\eta^{p+1}\right] .
$$

Then $\left\|\vec{u}_{0}\right\|_{X^{2}}+\left|\Lambda^{1} u_{0}\right|_{1}+\left|v_{0}\right|_{1}<\delta$ must imply $q(t)<\eta$ for all $t \geq 0$. Otherwise, by continuity of $q(t)$, we would have $q(t)=\eta$ for some $t$, and then (3.5) would contradict (3.4).

By Theorem 3.1, we are able to obtain a nonlinear scattering result for small solutions.

TheOREM 3.2. Let $\vec{u}(t)=(u(t), v(t))$ be the solution of (1.2) with initial data as in the previous theorem, $f(u)=u^{p+1}$ and $p>11$. Then there are $\vec{u}_{-}$and $\vec{u}_{+}$such that $\left\|\vec{u}(t)-\vec{u}_{ \pm}(t)\right\|_{X^{1}}$ tends to 0 as $t$ tends to $\pm \infty$, where $\vec{u}_{ \pm}(t)=S(t) \vec{u}_{ \pm}$solves the linear equation. 
Proof. Define

$$
\begin{aligned}
\vec{u}_{+}(t) & =S(t) \vec{u}_{0}+\int_{0}^{\infty} S(t-\tau) \partial_{x}\left(\begin{array}{c}
-\Lambda^{-2} f(u) \\
0
\end{array}\right) d \tau \\
& =\vec{u}(t)+\int_{t}^{\infty} S(t-\tau) \partial_{x}\left(\begin{array}{c}
-\Lambda^{-2} f(u) \\
0
\end{array}\right) d \tau .
\end{aligned}
$$

The function $\vec{u}_{+}(t)$ is a solution of the linear equation $(1.2)$, so $\vec{u}_{+}(t)=$ $S(t) \vec{u}_{+}$for some $\vec{u}_{+}$. It follows that

$$
\begin{aligned}
\left\|\vec{u}(t)-\vec{u}_{+}(t)\right\|_{X^{1}} & \leq \int_{t}^{\infty}\left\|S(t-\tau) \partial_{x}\left(\begin{array}{c}
-\Lambda^{-2} f(u) \\
0
\end{array}\right)\right\|_{X^{1}} d \tau \\
& =\int_{t}^{\infty}\left\|S(t-\tau) \partial_{x} \Lambda^{-2}\left(u^{p+1}\right)\right\|_{X^{1}} d \tau \\
& \leq c \int_{t}^{\infty}\left\|u^{p+1}(\tau)\right\|_{1} d \tau \leq c \int_{t}^{\infty}|u(\tau)|_{\infty}^{p}\|u\|_{1} d \tau \\
& \leq C\left\|\vec{u}_{0}\right\|_{1} \int_{t}^{\infty}(1+\tau)^{-p \theta} d \tau \leq C\left\|\vec{u}_{0}\right\|_{1}(1+t)^{1-p \theta} \rightarrow 0
\end{aligned}
$$

as $t \rightarrow \infty$, since in this case $\theta=-1 / 10$ and $1-p \theta<0$. In the estimate above we use the inequality $\|u\|_{1} \leq\|u\|_{1}+|v|_{2}=\left\|\vec{u}_{0}\right\|_{X^{1}}$.

The case $t \rightarrow-\infty$ involving $u_{-}$is completely analogous by the change of variables $t \mapsto-t$.

Acknowledgements. I would like to express my thanks to Prof. Piotr Biler for his useful advice and support.

\section{References}

[1] J. Albert, On the decay of solutions of the generalized Benjamin-Bona-Mahony equation, J. Math. Anal. Appl. 141 (1989), 527-537.

[2] - Dispersion of low-energy waves for the generalized Benjamin-Bona-Mahony equation, J. Differential Equations 63 (1986), 117-134.

[3] C. Amick, J. Bona and M. Schonbek, Decay of solutions of some nonlinear wave equations, ibid. 81 (1989), 1-49.

[4] L. Chen, Stability and instability of solitary waves for generalized symmetric regularized-long-wave equations, Phys. D 118 (1998), 53-68.

[5] J. Dziubański and G. Karch, Nonlinear scattering for some dispersive equations generalizing Benjamin-Bona-Mahony equation, Monatsh. Math. 122 (1996), 35-43.

[6] Y. Liu, Existence and blow up of solutions of a nonlinear Pochhammer-Chree equation, Indiana Univ. Math. J. 45 (1996), 797-816.

[7] C. Seyler and D. Fenstermacher, A symmetric regularized-long-wave equation, Phys. Fluids 27 (1984), 4-7. 
[8] P. Souganidis and W. Strauss, Instability of a class of dispersive solitary waves, Proc. Roy. Soc. Edinburgh Sect. A 114 (1990), 195-212.

Faculty of Mathematics and Informatics

Shumen University

9712 Shumen, Bulgaria

E-mail: shakkaev@fmi.shu-bg.net

Received on 13.4.2003;

revised version on 4.5.2004 